\title{
UTILIZATION OF ANTIBIOTICS AMONG PHYSICIANS IN THE GENERAL OUTPATIENT UNIT OF A TERTIARY HEALTH FACILITY IN NORTH CENTRAL NIGERIA
}

\author{
MO JAMIU ${ }^{1}$, A GIWA ${ }^{1}$, IK BELLO ${ }^{2}$
}

\begin{abstract}
Background: Antibiotics are prescribed to treat infections caused by suspected or confirmed organism(s). Inappropriate antibiotic prescribing could lead to treatment failure increase cases of toxicity and development of resistant strains of organism.
\end{abstract}

Objective: To assess the pattern of antibiotics prescribing among physician in the General Outpatients Department of University of Ilorin Teaching Hospital in Ilorin.

\begin{abstract}
Method: The study involved a 6 month retrospective review of prescription sheets in the General Outpatient Department of University of Ilorin Teaching Hospital (UITH) to assess the prescription pattern. A total of 3,552 prescriptions were reviewed. Information on prescribing pattern of physicians and patient demographics were obtained through a date collection form. The data obtained were entered into Statistical Package for Social Scientists (SPSS) version 16 and analyzed using descriptive value. Logistic regression was used in inferential statistic and P values Â 0.05 were considered significant.
\end{abstract}

\begin{abstract}
Results: The prescribing rate of antibiotic was $82.13 \%$. The most widely prescribed antibiotics were amoxicillin/ clavulanic acid (21.9\%), ciprofloxacin (21.2\%), metronidazole (17.2\%) and Amoxycillin (9.4\%). Average number of drugs per prescription was $4.32 \pm 1.46$. There was significant difference in the prescribing duration of antibiotics in female compare to male patient. Female patients had longer duration than their male counterpart.
\end{abstract}

Conclusion: There was high rate of antibiotic prescribing among physicians in the health facility. Rational prescribing in terms of generic prescribing and prescribing from Essential Drug List (EDL) was adequately complied with and the number of prescribed medications per prescription was within the limit.

Key words: Antibiotics; rational prescribing; amoxicillin-clavulanic; prescribing guideline.

Received: 21 December 2016

Accepted: 28 May 2017

\section{Introduction}

Drug utilization research is defined as a marketing, distribution, prescription, and use of drugs in society, with special emphasis on the resulting medical, social and economic consequences. ${ }^{1}$ Drug utilization includes the prescribing, dispensing, administering and ingestion of drugs. ${ }^{2}$ A drug utilization study is a structured process which is used to assess the quality of drug therapy by engaging in the evaluation of data on drug prescribing, dispensing and patient use in a given health care environment, against predetermined, agreed upon

1. Department of Clinical Pharmacy and Pharmacy Practice, Faculty of Pharmaceutical Sciences, University of Ilorin, Ilorin, Nigeria

2. Pharmacy department, University of Ilorin Teaching Hospital, Ilorin, Nigeria

Address of Correspondence: Jamiu M.O. jamolakunle@gmail.com +234-07039027492 criteria and standards, with special emphasis on the resulting medical, social, and economic consequences. It seeks to monitor, evaluate and suggest modifications in the prescribing practices with the aim of making the medical care rationale and cost effective. ${ }^{3}$ The Drug Utilization 90\% (DU 90\%) index is a simple, inexpensive and flexible method for assessing the quality of the drug prescription. ${ }^{1}$

Antimicrobial resistance is an important patientsafety and public-health issue. The number of infections due to antibiotic-resistant bacteria is growing and the pipeline of new classes of antibiotics is very limited. The emergence of antibiotic resistance is linked to antimicrobial exposure, and the overall uptake of antibiotics in a population, as well as the way antibiotics are used by individuals has been shown to affect antibiotic resistance. The results of a recent meta-analysis have found strong evidence linking the prescribing of antibiotics in primary care

Bangladesh J Medicine 2017; $28: 64-69$ 
to antimicrobial resistance in pathogens causing urinary, respiratory and skin infections in individual patients. The likelihood of isolating resistant bacteria from a patient is strongly associated with the number and duration of antibiotic courses prescribed in the previous 12 months. ${ }^{4}$ This study therefore seek to assess the utilization of antibiotics among the physicians through prescribing pattern and conformity with World Health Organization(WHO ) guideline for good prescribing practice in the tertiary Health Facility between January, 2016 and December, 2016.

\section{Study Methods}

This study involved 1-year retrospective review of antibiotic prescription sheets at the General Outpatient Department of the University of Ilorin Teaching Hospital (UITH) between January and December, 2016. UITH is a tertiary heath facility with 650 bed space located at Oke-Oyi in Ilorin East local Government area of Kwara State. All Prescriptions for adult male and female containing at-least one antibiotic within the study period were selected for the study. All prescriptions selected were assessed from patient's identifiers and prescriber's identifiers to ensure validity of the prescriptions.

Designed data collections forms were used to collect necessary information. Data obtained were entered into excel spread sheet and Statistical Package for Social Sciences was used to analyze the data. Results were presented descriptively in the form of tables and charts where applicable. P-value less than 0.5 were considered statistically significant.

\section{Results}

A total of 4, 325 prescriptions were used, out of which 3,552 contained at least one antibiotic. This gives a prescribing rate of $82.13 \%$. The total number of the antibiotics in these prescriptions was found to be 3882. The pattern of antibiotics prescribing among the physicians was as shown in the table (Table-I).
Table-I

Frequency of antibiotics prescribed by their generic names

\begin{tabular}{lc}
\hline Name of antibiotic & Frequency $(\%) \mathrm{N}=3882$ \\
\hline Amoxicillin/ clavulanic acid & $846(21.9)$ \\
Amoxicillin & $366(9.4)$ \\
Ampicillin/sulbactam & $12(0.3)$ \\
Ampicillin & $6(0.2)$ \\
Ampiclox & $6(0.2)$ \\
Azithromycin & $144(2.9)$ \\
Cefixime & $48(1.2)$ \\
Cefuroxime & $192(5.1)$ \\
Cephalexin & $6(0.2)$ \\
Ciprofloxacin/tinidazole & $138(3.6)$ \\
Ciprofloxacin & $822(21.2)$ \\
Clarithromycin & $42(1.1)$ \\
Cotrimoxazole & $30(0.8)$ \\
Doxycycline & $120(3.1)$ \\
Erythromycin & $144(3.7)$ \\
Fluconazole & $96(2.5)$ \\
Gentamicin & $6(0.2)$ \\
Ketoconazole & $6(0.2)$ \\
Levofloxacin & $72(1.9)$ \\
Metronidazole & $666(17.2)$ \\
Neomycin & $6(0.2)$ \\
Nitrofurantoin & $18(0.5)$ \\
Ofloxacin & $12(0.3)$ \\
Secnidazole & $42(1.1)$ \\
Sparfloxacin & $24(0.6)$ \\
Tetracycline & $30(0.8)$ \\
Tinidazole & $12(0.3)$ \\
\hline &
\end{tabular}

When the prescriptions were analyzed for WHO standards for good prescribing practice, the following results were obtained (Table-II).

Results obtained from average number of medications per prescription indicated that the number of medications per prescription per encounter showed that the most frequently occurring number of medications per prescription was five (five medications per prescription) while ten medications per prescription occurred the least (Figure-1).

Table-II

The characteristics of the prescriptions analyzed

\begin{tabular}{lll}
\hline Variables & Options & Frequency $(\%) \mathrm{N}=3882$ \\
\hline Generic prescription & Generic & $2190(56.4)$ \\
& Branded & $1692(43.6)$ \\
Dosage form & Tablets & $3126(80.5)$ \\
& Capsules & $750(19.3)$ \\
Availability in EDL & Injectable & $6(0.2)$ \\
Availability for dispensing & Available & $3546(91.3)$ \\
\hline
\end{tabular}

*EDL=Essential Drug List 
Table-III

Logistic regression comparing the use of antibiotics among male and female patients

\begin{tabular}{lcccc}
\hline Duration of prescribed antibiotics & Odd Ratio & Z & P-value & 95\% CI of OR \\
\hline Less than 3 days & 0.246 & 7.477 & 0.006 & $0.253-0.298$ \\
Between 3 and 7 days & 0.269 & 21.351 & 0.000 & $0.154-0.470$ \\
More than 7 days & 0.661 & 5.125 & 0.024 & $0.463-0.946$ \\
\hline
\end{tabular}

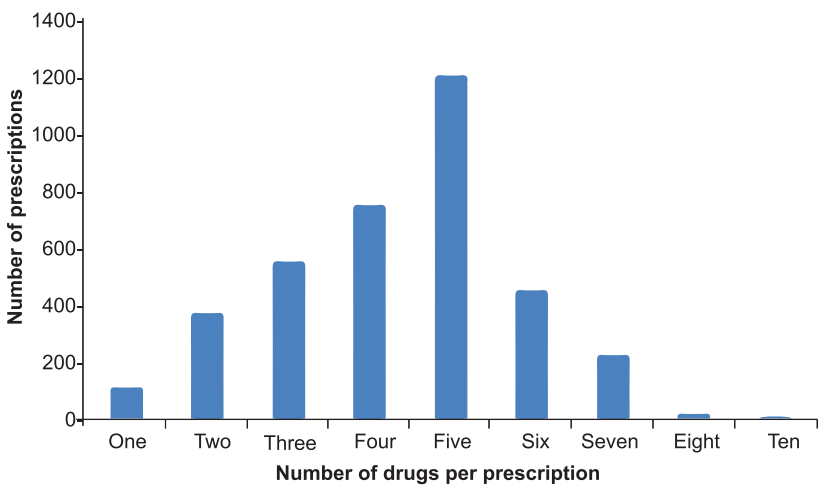

Fig.-1: Description of number of prescribed medications per prescription containing antibiotics

The duration of prescribing of antibiotics revealed that most of the antibiotics were prescriber for between 5 to 10 days. Longer duration for over thirty days was minimal which accounted for less than $1 \%(0.31 \%)$. See the figure bellow (Figure 2).

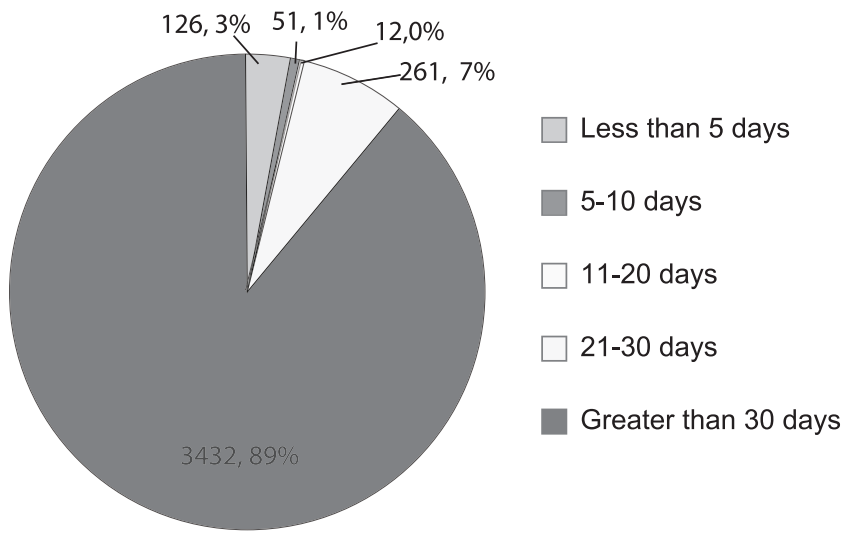

Fig.-2: Description of duration of prescribed antibiotics

Logistic regression analysis to compare the duration of prescribed antibiotics between male and female patients indicated that there was gender influence in shorter course of antibiotics use. Male patients were received more short courses on antibiotics (1-7 days) than their female counterpart whereas both were not equally different for longer course of treatment (Table-III).

\section{Discussion}

A prescription provides an insight into a prescriber's attitude to the disease being treated, the nature of healthcare delivery system in the community and the treatment options available. ${ }^{5}$ The prescribing patterns of physicians in the institution understudy indicated that more than three quarter of prescriptions contained one or more antibiotics. More than half of these were prescribed in generic which was fairly good practice according to World Health Organization Guideline. ${ }^{6}$ Tablet was the most frequently prescribed dosage form. This was followed by capsules and with injectable least prescribed. Most of prescribed antibiotics were from essential drug list which was a good prescribing practice in accordance with WHO specification. Only a fraction of prescribed antibiotics were not available for dispensing, less than 10\%. A better result was obtained from a study in Federal Staff Clinic, Abuja where $97.9 \%$ of prescribed antibiotics were available for dispensing. ${ }^{7}$

The most commonly prescribed antibiotics were Amoxycillin/clavulanic acid and ciprofloxacin and this was similar to a study in Turkey, where Amoxicillin/ clavulanic acid was the most commonly prescribed with $18.1 \%$ prescribing rate close to the rate obtained in this study (21.9\%). Ciprofloxacin was also found to be most commonly prescribed antibiotic in a similar study carried out in Sokoto, North-Central Nigeria. ${ }^{8}$ This was contrary to the results obtained by Abusaeed et al, 2013 and Widayaty et al 2011 where Amoxycillin was found to be the most widely prescribed antibiotic. Metronidazole was next to the two mentioned drugs followed by amoxicillin. Others were cefuroxime, erythromycin, ciprofloxacin-tinidazole combination, doxycycline, and fluconazole. The least prescribed were ampicillin, Ampicillin-cloxacillin and cephalexin.

The most occurring number of medications per prescription was five medications per prescription while those prescriptions containing up to ten medications were the least (Figure 1). This suggests a less tendency for polypharmacy in the medication use because majority of the patients were on the average of five medications per prescription. The lower the numbers of drugs taken by the patient the less 
the chance for interaction. ${ }^{9}$ The more drugs a patient is exposed to, the more likely they are to be prescribed inappropriately. ${ }^{10,11}$

There was a statistically significant difference between pattern of antibiotic prescribing between male and female patients. Female patients are more likely to be prescribed antibiotics for short (less than three days) and medium (up to seven days) courses of therapy. This may not be unconnected with the fact that some antibiotics that are used in short course e.g Tinidazole and Fluconazole are indicated for therapy in Sexually Transmitted Diseases like Chlamydia Trichomoniasis and candidiasis in which female have been found to be more affected than their male counterpart. ${ }^{12}$ Also, Urinary Tract Infections occur more often in women than men at a ratio of $8: 1$. Approximately $50-60 \%$ of women report at least one UTI in their lifetime and one in three will have at least one symptomatic UTI necessitating antibiotic treatment by age $24 .{ }^{13}$ On the other hand, both male and female are likely to be equally affected with respiratory tract infections. While male are more commonly affected by otitis media, croup and lower respiratory infections, female are more commonly affected by upper respiratory infections like like tonsillitis, sinusitis and otitis externa. This could probably be the basis for no gender difference in antibiotics prescribing for long duration since these ailments require long duration of antibiotic use. ${ }^{14}$

Antibiotics are prescribed more by emergency physicians and family medicine practitioners. Patients who are seen in the emergency room and in the family practitioner's office are more likely to receive antibiotic prescriptions. ${ }^{8}$

Although many countries have been successful in reducing primary care prescribing of antimicrobials, primary care is still responsible for the majority of antibiotics prescribed to people. Much of this use is in the treatment of suspected respiratory infection and levels of prescribing vary widely within and between countries, suggesting that further reductions are possible. However, there are many barriers to reducing the inappropriate use of antimicrobials, including patient and practitioner expectations. ${ }^{15}$

Antibiotic prescribing practices vary widely and errors are common. About half of patients receive an antibiotic for at least one day during the course of an average hospital stay. The most common types of infections for which hospital clinicians wrote antibiotic prescriptions were lung infections (22\%), urinary tract infections (14\%), and suspected infections caused by drug-resistant Staphylococcus bacteria, such as Methicinllin Reasistant Staphylococcus aureus (17\%). About 1 out of 3 times, prescribing practices to treat urinary tract infections and prescriptions for the critical and common drug vancomycin included a potential error - given without proper testing or evaluation, or given for too long. Doctors in some hospitals prescribed up to 3 times as many antibiotics as doctors in similar areas of other hospitals. This difference suggests the need to improve prescribing practices. ${ }^{16}$

Poor prescribing puts patients at risk. Although antibiotics save lives (for example, in the prompt treatment of sepsis, a life-threatening infection throughout the body), they can also put patients at risk for a Clostridium difficile infection, deadly diarrhea that causes at least 250,000 infections and 14,000 deaths each year in hospitalized patients. Decreasing the use of antibiotics that most often lead to $C$. difficile infection by $30 \%$ (this is $5 \%$ of overall antibiotic use) could lead to $26 \%$ fewer of these deadly diarrheal infections. These antibiotics include fluoroquinolones, ${ }^{2}$-lactams with ${ }^{2}$ dlactamase inhibitors, and extended-spectrum cephalosporins. Patients getting powerful antibiotics that treat a broad range of infections are up to 3 times more likely to get another infection from an even more resistant germ.

Observational studies have shown that physicians who provide samples to their patients are less likely to recommend over-the-counter medications, less likely to prescribe preferred medications and more likely to prescribe advertised medications. ${ }^{17}$

Various drug prescription problems have been identified in health facilities in developing countries like Nigeria. This includes irrational use of drugs, unnecessary polypharmacy and high use of drugs with unproven efficacy. Some of these studies have shown that antibiotics are one of the most commonly prescribed drugs among physicians and have identified its overuse. ${ }^{7}$

One study has also revealed characteristic behavior of doctors and patients and laxity in regulation for prescribing and dispensing antibiotics. Doctorrelated factors include diagnostic uncertainty especially in distinguishing between viral and bacterial infections especially in cases of upper respiratory tract infections and diarrhea. To deal with the uncertainty about whether or not the infection is bacterial, they prescribe antibiotics. Even in cases where patients report fever for a day or two, it was difficult to ascertain the cause.

Moreover, medical tests were not recommended to remove the ambiguity as most patients did not want to take a test for a day's fever or could not afford to 
take these tests due to lack of time or money. As a result, antibiotics are prescribed where they are not required. Generally, in public primary health care facilities, microbiology lab facilities are not available and most of the patients who visit these facilities cannot afford the tests from private labs. So doctors relied mainly on their clinical acumen.

Another factor identified was doctors' perceived demand and expectation. Doctors from both private and public sectors said that some patients demand capsules and 'strong' medicines. Some patients who had previously been prescribed antibiotics asked to be given these drugs again. Educated patients may also demand for antibiotics that they wanted. At times, doctors often succumb to patients' demand or expectations for antibiotic prescription. ${ }^{18}$

Many doctors said that the patient expects them to write a prescription for antibiotics when they have spent money on consulting a doctor or waited for considerable time in a public facility. Patients are not satisfied if they get just paracetamol or oral rehydration solution packets (ORS) for fever and diarrhoea, which they could have taken on their own; doctors feel patients want them to prescribe something that they have not taken and that is why doctors prescribe antibiotics. ${ }^{18}$

The use of trade names was also found to be very rampant among the prescribers; this may not be unconnected with the immense pressure which was mounted by the pharmaceutical company representatives on the prescribers and the frequent inducement or entertainment in the form of gifts. ${ }^{8}$ Several health professionals have issued statements to reduce the use of antibiotics worldwide by means of strict antibiotics policy. For such policy to be implemented, detailed knowledge of antibiotics prescribing pattern and related cost is important. ${ }^{19}$ Generic prescribing has also been identified as a key factor in reducing the cost of treatment as generic drug are less expensive than branded ones. ${ }^{20}$

\section{Acknowledgment}

The authors acknowledge the staff and management of University of Ilorin Teaching Hospital and the pharmacy department in particular for their cooperation.

\section{References}

1. World Health Organization. Promoting Rational Use of Medicines: Core Components-WHO Policy perspectives on medicine. 2003.. Essential medicines and Health Products Information Portal. A World Health Organization Resource. [Last accessed on 2014 Apr 22]. Available from:http:// www.apps.who.int/medicinedocs/en/d/Jh3011e/ 2.html .

2. Serradell J, Bjornson DC \& Hartzema AG. Drug Utilization Study Methodologies: National and International Perspectives. Drug Intelligence and Clinical Pharmacy. 1987; 21: 994-1001.

3. Jain S, Upadhyaya P, Goyal J, Kumar A, Jain P, Seth V et al (2015). A systematic review of prescription pattern monitoring studies and their effectiveness in promoting rational use of medicines. Perspect Clin Res 2015; 6(2): 86-90.

4. Ashiru-Orelope D, Sharland M,Charani E, McNalty C, Cooke J.( Improving the quality of antibiotic prescribing in the NHS by developing a new antimicrobial stewardship program: start smart-then focus. J antimicrobial chemotherapy 2012; 65 Suppl. 1:51-63.

5. Adegoke OO, Pongri A,Nkpubre G. (2014) Review of two year prescription patterns of antimalarial drug regimen at NIPRD Health Centre, Abuja. West African Journal of Pharmacy2014; 25(1)88-96.

6. WHO. Introduction to drug utilization research. WHO Library Cataloguing-in-Publication Data 2003; 1-49. Available online at http://apps.who.int/ medicinedocs / pdf/s 4876 e/s4876e.pdf. Last assessed on the $10^{\text {th }}$ April 2017.

7. Abu-Saeed K, Joseph GS, Joseph FL. Prescription Pattern of Antibiotics among Physicians in a Secondary Health Facility in Abuja, Nigeria. British Journal of Pharmaceutical Research 2013; 3(4): 940-7.

8. Jimoh AO, Etuk EU, Sanni Z, Shuaibu HA. The Pattern of Antibiotic Use in a Family Medicine Department of a Tertiary Hospital in Sokoto, North Western Nigeria. Journal of Clinical and Diagnostic Research 2011; 5(3): 566-9.

9. Salazar JA, Poon I, Nair M. Clinical consequences of polypharmacy in the elderly: expect the unexpected, think the unthinkable. Expert Opin Drug Saf 2007;6: 695-704.

10. Hilmer SN, Gnjidic D. The Effects of Polypharmacy in Older Adults. Clinical Pharmacology \& Therapeutics. $1^{\text {st }}$ ed. 2008, 86-88 doi:10.1038/clpt. 2008.224 .

11. Lin P. Drug interactions and polypharmacy in elderly. The Canadian Alzeimer Disease Review 2003; 10-14.

12. United States Centre for Disease control (US/CDC). Trends in Reportable Sexually Transmitted Diseases in the United States 2014. Available online at http:/ /www.cdc.gov/std/stats05/trends2005.htm.

13. Al-Badr A, Al-Shaikh G. Recurrent Urinary Tract Infections Management in Women. Sultan Qaboos Univ Med J 2013; (3): 359-67. 
14. Wong MD, Blumberg AD, Lowe, GL. Guidelines for the Use of Antibiotics in Acute Upper Respiratory Tract Infections. Am Fam Physician 2006; 74(6): 956-66.

15. Metcalfe C, Lovering A, Mant D. Effect of antibiotic prescribing in primary care on antimicrobial resistance in individual patient: systematic review and meta- analysis. BMJ. 2010; 340:c2096 doi:10.1136.

16. United States Centre for Disease Control and Prevention. Antibiotic resistance threats in the United States 2013. Available online at https:// www.cdc.gov/drugresistance/pdf/ar-threats-2013508.pdf. Last assessed on the $15^{\text {th }}$ March, 2016.
17. Pinckney RG, Helminski AS, Kennedy AG, Maclean $\mathrm{CD}$, Hurowitz L, Cote E. The effect of medication samples on self- reported prescribing practices: A state wide, cross sectional survey. J Gen Intern Med 2010; 26(1): 40-5.

18. Kotwani A, Wattal C, Katewa S, Joshi PC, Holloway K. Factors influencing primary care physicians to prescribe antibiotics in Delhi India. Family Practice. 2010 27:684-690.

19. Akande TM, Ologe M, Medubi GF. Antibiotic prescription pattern and cost at University of Ilorin Teaching Hospital, Ilorin, Nigeria. International Journal of Tropical Medicine 2009; 4(2): 50-4.

20. Giwa A, Tayo.F. Poverty affordability of antibiotics drugs and glycemic control: An unholy alliance to a developing economy. International Journal of Pharmaceutical Sciences and Research 2013; 4(8): 113-20. 